\title{
OPTIMALITY CONDITIONS AND DUALITY RESULTS FOR A NEW CLASS OF NONCONVEX NONSMOOTH VECTOR OPTIMIZATION PROBLEMS
}

\author{
TADEUSZ ANTCZAK AND RAM VERMA
}

Received 08 December, 2018

\begin{abstract}
In this paper, a new class of nonconvex nonsmooth multiobjective programming problems with both inequality and equality constraints defined in a real Banach space is considered. Under the nondifferentiable vectorial $(\Phi, \rho)^{w}$-invexity notion introduced in the paper, optimality conditions and duality results in Mond-Weir sense are established for the considered nonsmooth vector optimization problem. It turns out that the results developed here under $(\Phi, \rho)^{w}$-invexity are applicable for a larger class of nonconvex nondifferentiable multiobjective programming problems than under several generalized convexity notions existing in the literature.
\end{abstract}

2010 Mathematics Subject Classification: 90C29; 90C46; 90C26; 49J52

Keywords: nonsmooth mutiobjective programming, efficient solution, nonsmooth $(\Phi, \rho)^{w}$-invexity, optimality conditions, Mond-Weir duality

\section{INTRODUCTION}

The term multiobjective programming (or vector optimization) is used to denote a type of optimization problems where two or more objectives are to be minimized subject to certain constraints. Investigation on sufficiency of (weak) Pareto optimality and duality has been one of the most attraction topics in the theory of multi-objective problems. This is a consequence of the fact that vector optimization problems are useful mathematical models of most real-life problems in economics, physics, mechanics, decision making, game theory, engineering, optimal control, etc. It is well known that the concept of convexity and its various generalizations play an important role in deriving sufficient optimality conditions and duality results for multiobjective programming problems. In recent years, therefore, multiobjective programming has grown remarkably in different directions in the settings of optimality conditions and duality theory. It has been enriched by the applications of various types of generalizations of convexity theory, with and without differentiability assumptions (see, for example, $[1,2,4,5,7,8,11,13-16,18,20,21]$ and others).

The aim of the present work is to introduce a new concept of nondifferentiable generalized invexity notion and to use it to prove optimality and duality results for 
a new class of nonsmooth multiobjective programming problems defined in a real Banach space. By taking the motivation from Antczak and Stasiak [6] and Stefanescu and Stefanescu [22], we introduce the concept of nondifferentiable $(\Phi, \rho)^{w}$-invexity for a nonsmooth multiobjective programming problem in which every component of functions involved is a locally Lipschitz function. However, the central purpose of this paper is to discuss application of the introduced vectorial nondifferentiable $(\Phi, \rho)^{w}$-invexity notion in proving the optimality results for a new class of nonconvex nondifferentiable multiobjective programming problems. Namely, we prove Karush-Kuhn-Tucker necessary optimality conditions for a (weak) Pareto optimal solution in the considered nondifferentiable multiobjective programming problem in which constraint functions are $(\Phi, \rho)^{w}$-invex. Sufficiency of these necessary optimality conditions for both weak Pareto and Pareto solutions is established for the class of constrained vector optimization problems with nondifferentiable $(\Phi, \rho)^{w}$-invex functions, not necessarily, with respect to the same $\rho$. Further, under $(\Phi, \rho)^{w}$-invexity hypotheses, several duality results are established between the considered nonsmooth multiobjective programming problem and its nondifferentiable vector dual problem in the sense of Mond-Weir. The optimality results proved in the paper are illustrated by an example of a nonconvex nonsmooth vector optimization problem involving nondifferentiable $(\Phi, \rho)^{w}$-invex functions.

\section{PRELIMINARIES}

Throughout this paper, we use the following conventions for vectors $x=$ $\left(x_{1}, x_{2}, \ldots, x_{n}\right)^{T}, y=\left(y_{1}, y_{2}, \ldots, y_{n}\right)^{T}$ in the Euclidean space $R^{n}$ :

(i) $x=y$ if and only if $x_{i}=y_{i}$ for all $i=1,2, \ldots, n$;

(ii) $x>y$ if and only if $x_{i}>y_{i}$ for all $i=1,2, \ldots, n$;

(iii) $x \geqq y$ if and only if $x_{i} \geqq y_{i}$ for all $i=1,2, \ldots, n$;

(iv) $x \geq y$ if and only if $x \geqq y$ and $x \neq y$.

In this section, we provide some definitions and some results that we shall use in the sequel. Throughout this paper, we denote a real Banach space by $X$, the (continuous) dual of $X$ by $X^{*}$, and the value of the function $\xi$ in $X^{*}$ at $v \in X$ by $\langle\xi, v\rangle$.

Definition 1 ([9]). The Clarke generalized directional derivative of a locally Lipschitz function $f: X \rightarrow R$ at $x \in X$ in the direction $v \in X$, denoted by $f^{0}(x ; v)$, is given by

$$
f^{0}(x ; v)=\limsup _{\substack{y \rightarrow x \\ \lambda \downarrow 0}} \frac{f(y+\lambda v)-f(y)}{\lambda} .
$$

Definition 2 ([9]). The Clarke generalized subgradient of a locally Lipschitz function $f: X \rightarrow R$ at $x \in X$, denoted by $\partial f(x)$, is defined as follows

$$
\partial f(x)=\left\{\xi \in X^{*}: f^{0}(x ; v) \geq\langle\xi, v\rangle \text { for all } v \in X\right\} .
$$

Let $S$ be a nonempty convex subset of $X$. 
Definition 3. The function $\Phi: S \rightarrow R$ is said to be quasi-convex if, for each $\alpha \in R$, the level set $\{x \in S: \Phi(x) \leqq \alpha\}$ is convex, or equivalently, if $\Phi(\lambda y+(1-\lambda) x) \leqq$ $\max \{\Phi(y), \Phi(x)\}$ for every $y, x \in S$ and $\lambda \in[0,1]$.

A stronger property is also considered as follows:

Definition 4. The function $\Phi: S \rightarrow R$ is said to be strictly quasi-convex if it is quasi-convex and $\Phi(\lambda y+(1-\lambda) x)<0$, whenever $\Phi(y)<0, \Phi(x) \leqq 0$ and $\lambda \in$ $(0,1)$.

Proposition 1. If $\Phi: S \rightarrow R$ is a strictly quasi-convex function and there are $x^{1}, \ldots, x^{k} \in S$ such that $\Phi\left(x^{i}\right) \leqq 0, i=1, \ldots, k$ and $\Phi\left(x^{i^{*}}\right)<0$ for at least one $i^{*} \in$ $\{1, \ldots, k\}$, then $\Phi\left(\sum_{i=1}^{k} \lambda_{i} x_{i}\right)<0$ for every $\lambda=\left(\lambda_{1}, \ldots, \lambda_{k}\right) \geq 0$ such that $\sum_{i=1}^{k} \lambda_{i}=1$ and $\lambda_{i^{*}}>0$.

In [22], Stefanescu and Stefanescu introduced the definition of a differentiable $(\Phi, \rho)^{w}$-invex function. Now, in the natural way, we generalize this definition to the nondifferentiable vectorial case.

Definition 5. Let $f=\left(f_{1}, \ldots, f_{k}\right): X \rightarrow R^{k}$ be defined on $X$, every its component $f_{i}, i=1, \ldots, k$, be a locally Lipschitz function on $X$ and $u \in X$. If there exist a function $\Phi: X \times X \times X^{*} \times R \rightarrow R$, where $\Phi(x, u,(\cdot, \cdot))$ is strictly quasi-convex on $X^{*} \times R$, $\Phi(x, u,(0, a)) \geqq 0$ for all $x \in X$ and each $a \in R_{+}$and $\rho=\left(\rho_{1}, \ldots, \rho_{k}\right) \in R^{k}$, where $\rho_{i}$, $i=1, \ldots, k$, are real numbers such that the following inequalities

$$
f_{i}(x)-f_{i}(u) \geqq \Phi\left(x, u,\left(\xi_{i}, \rho_{i}\right)\right), i=1, \ldots, k \quad(>)
$$

hold for all $x \in X(x \neq u)$ and each $\xi_{i} \in \partial f_{i}(u), i=1, \ldots, k$, then $f$ is said to be a nonsmooth vector $(\Phi, \rho)^{w}$-invex at $u$ on $X$. If inequalities (2.1) are satisfied at any point $u$, then $f$ is said to be a nonsmooth (strictly) vector $(\Phi, \rho)^{w}$-invex function on $X$.

In order to define an analogous class of nondifferentiable vector (strictly) $(\Phi, \rho)^{w}$ incave functions, the direction of the inequality in the definition of these functions should be changed to the opposite one.

Remark 1. Let $X \subseteq R^{n}$ and the functional $\Phi(x, u,(\cdot, \cdot))$ be convex on $R \times R$. From Definition 5, there are the following special cases:

a) If $\Phi\left(x, u,\left(\xi_{i}, \rho_{i}\right)\right)=\left\langle\xi_{i}, x-u\right\rangle$, where $\xi_{i} \in \partial f_{i}(u), i=1, \ldots, k$, then we obtain the definition of a nondifferentiable convex function.

b) If $\Phi\left(x, u,\left(\xi_{i}, \rho_{i}\right)\right)=\left\langle\xi_{i}, \eta(x, u)\right\rangle$ for a certain mapping $\eta: X \times X \rightarrow R^{n}$, where $\xi_{i} \in \partial f_{i}(u), i=1, \ldots, k$, then we obtain the definition of a locally Lipschitz invex function (with respect to the function $\eta$ ) (see Lee [16] and Kim and Schaible [14] in a nonsmooth vectorial case).

c) If $\Phi\left(x, u,\left(\xi_{i}, \rho_{i}\right)\right)=\frac{1}{b_{i}(x, u)}\left\langle\xi_{i}, \eta(x, u)\right\rangle$, where $b_{i}: X \times X \rightarrow R_{+} \backslash\{0\}$ and $\eta: X \times X \rightarrow R^{n}$, then we obtain the definition of a nondifferentiable $b$-invex 
function (with respect to the function $\eta$ ) (see, Li et al. [17] in a nondifferentiable scalar case).

d) If $\Phi\left(x, u,\left(\xi_{i}, \rho_{i}\right)\right)=\left\langle\xi_{i}, x-u\right\rangle+\rho_{i}\|x-u\|^{2}$, then $(\Phi, \rho)^{w}$-invexity reduces to the definition of a nonsmooth $\rho$-convex function (see Zalmai [23]).

e) If $\Phi\left(x, u,\left(\xi_{i}, \rho_{i}\right)\right)=\left\langle\xi_{i}, \eta(x, u)\right\rangle+\rho_{i}\|\theta(x, u)\|^{2}$ for a certain mapping $\eta: X \times X \rightarrow R^{n}$, where $\theta: X \times X \rightarrow R^{n}, \theta(x, u) \neq 0$, whenever $x \neq u$, then $(\Phi, \rho)^{w}$-invexity reduces to the definition of a nonsmooth $\rho$-invex function (with respect to $\eta$ and $\theta$ ) introduced by Jeyakumar [12] in a scalar case.

f) If $\Phi\left(x, u,\left(\xi_{i}, \rho_{i}\right)\right)=\alpha_{i}(x, u)\left\langle\xi_{i}, \eta(x, u)\right\rangle$, where $\alpha_{i}: X \times X \rightarrow R_{+} \backslash\{0\}$, $\theta: X \times X \rightarrow R^{n}, \theta(x, u) \neq 0$, whenever $x \neq u$, then $(\Phi, \rho)^{w}$-invexity reduces to the definition of a $V$-invex function (with respect to $\eta$ ) introduced by Jeyakumar and Mond [13] in a differentiable case and Mishra and Mukherjee [19] in a nonsmooth case.

g) If $\Phi\left(x, u,\left(\xi_{i}, \rho_{i}\right)\right)=\alpha_{i}(x, u)\left\langle\xi_{i}, \eta(x, u)\right\rangle+\rho_{i}\|\theta(x, u)\|^{2}$, where $\alpha_{i}: X \times X \rightarrow$ $R_{+} \backslash\{0\}, \theta: X \times X \rightarrow R^{n}, \theta(x, u) \neq 0$, whenever $x \neq u$, then $(\Phi, \rho)^{w}$-invexity reduces to the definition of a nonsmooth $V$ - $\rho$-invex function (with respect to $\eta$ and $\theta$ ) introduced by Kuk et al. [15].

h) If $\Phi\left(x, u,\left(\xi_{i}, \rho_{i}\right)\right)=F\left(x, u, \xi_{i}\right)$, where $F(x, u, \cdot)$ is a sublinear functional with respect to the third component, then the definition of a $(\Phi, \rho)^{w}$-invex function reduces to the definition $F$-convexity introduced by Hanson and Mond [10].

i) If $\Phi\left(x, u,\left(\xi_{i}, \rho_{i}\right)\right)=F\left(x, u, \xi_{i}\right)+\rho_{i} d^{2}(x, u)$, where $F(x, u, \cdot)$ is a sublinear functional with respect to the third component and $d: X \times X \rightarrow R$ is a pseudometric on $X$, then the definition of a $(\Phi, \rho)^{w}$-invex function reduces to the definition $(F, \rho)$-convexity introduced in a nondifferentiable case by Bhatia and Jain [8] .

j) If the functional $\Phi(x, u,(\cdot, \cdot))$ is convex on $X^{*} \times R$, then we obtain the definition of a nondifferentiable $(\Phi, \rho)$-invex function (see Antczak and Stasiak [6] in a scalar case).

\section{OPTIMALITY UNDER NONSMOOTH $(\Phi, \rho)^{w}$-INVEXITY}

In the paper, we consider the following nonsmooth vector optimization problem:

$$
\begin{gathered}
f(x):=\left(f_{1}(x), \ldots, f_{k}(x)\right) \rightarrow V \text {-min } \\
\text { subject to } \quad g_{j}(x) \leqq 0, j \in J=\{1, \ldots m\}, \\
h_{t}(x)=0, t \in T=\{1, \ldots, q\}, \\
x \in X,
\end{gathered}
$$

where $f_{i}: X \rightarrow R, i \in I=\{1, \ldots, k\}, g_{j}: X \rightarrow R, j \in J$, and $h_{t}: X \rightarrow R, t \in T$ are locally Lipschitz functions on $X$. For the purpose of simplifying our presentation, we will next introduce some notations which will be used frequently throughout this paper. Let $D:=\left\{x \in X: g_{j}(x) \leqq 0, j \in J, h_{t}(x)=0, t \in T\right\}$ be the set of all feasible 
solutions in problem (VP), and $J(\bar{x})$ be a set of active inequality constraints at point $\bar{x} \in D$, that is, $J(\bar{x})=\left\{j \in J: g_{j}(\bar{x})=0\right\}$.

Definition 6. A feasible point $\bar{x}$ is said to be a weak Pareto solution (weakly efficient solution, weak minimum) for (VP) if and only if there exists no other $x \in D$ such that $f(x)<f(\bar{x})$.

Definition 7. A feasible point $\bar{x}$ is said to be a Pareto solution (efficient solution) for (VP) if and only if there exists no other $x \in D$ such that $f(x) \leq f(\bar{x})$.

Theorem 1 (Generalized F. John necessary optimality conditions, [9]). Let $\bar{x} \in D$ be a (weakly) efficient solution of the considered nonsmooth multiobjective programming problem (VP). Then, there exist $\bar{\lambda} \in R^{k}, \bar{\mu} \in R^{m}$ and $\bar{\vartheta} \in R^{q}$ such that

$$
\begin{gathered}
0 \in \sum_{i=1}^{k} \bar{\lambda}_{i} \partial f_{i}(\bar{x})+\sum_{j=1}^{m} \bar{\mu}_{j} \partial g_{j}(\bar{x})+\sum_{t=1}^{q} \bar{\vartheta}_{t} \partial h_{t}(\bar{x}), \\
\bar{\mu}_{j} g_{j}(\bar{x})=0, j \in J, \\
(\bar{\lambda}, \bar{\mu}) \geq 0 .
\end{gathered}
$$

Now, we prove the so-called Generalized Karush-Kuhn-Tucker necessary optimality conditions for a nonsmooth vector optimization problem with locally Lipschitz $(\Phi, \rho)^{w}$-invex constraint functions.

Theorem 2 (Generalized Karush-Kuhn-Tucker necessary optimality conditions). Let $\bar{x} \in D$ be a (weakly) efficient solution of the considered multiobjective programming problem (VP) and Generalized F. John necessary optimality conditions (3.1)(3.3) be satisfied at $\bar{x}$ with Lagrange multipliers $\bar{\lambda} \in R^{k}, \bar{\mu} \in R^{m}$ and $\bar{\vartheta} \in R^{q}$. Further, assume that there exists a feasible solution $\widetilde{x}$ such that $g_{j}(\widetilde{x})<0, j \in J(\bar{x})$ and, moreover, $g_{j}, j \in J(\bar{x})$, is locally Lipschitz $\left(\Phi, \rho_{g_{j}}\right)^{w}$-invex at $\bar{x}$ on $D, h_{t}, t \in T^{+}(\bar{x}):=$ $\left\{t \in T: \bar{\vartheta}_{t}>0\right\}$, is locally Lipschitz $\left(\Phi, \rho_{h_{t}}\right)^{w}$-invex at $\bar{x}$ on $D-h_{t}, t \in T^{-}(\bar{x}):=$ $\left\{t \in T: \bar{\vartheta}_{t}<0\right\}$, is locally Lipschitz $\left(\Phi, \rho_{h_{t}}\right)^{w}$-invex at $\bar{x}$ on $D$ and $\sum_{j=1}^{m} \bar{\mu}_{j} \rho_{g_{j}}+$ $\sum_{t \in T^{+}(\bar{x})} \bar{\vartheta}_{t} \rho_{h_{t}}^{+}-\sum_{t \in T^{-}(\bar{x})} \bar{\vartheta}_{t} \rho_{h_{t}}^{-} \geqq 0$. Then $\bar{\lambda} \neq 0$.

Proof. Let $\bar{x} \in D$ be an efficient (weakly efficient) solution of the considered multiobjective programming problem (VP). Then, the necessary optimality conditions of F. John type (3.1)-(3.3) are fulfilled with the Lagrange multipliers $\bar{\lambda} \in R^{k}, \bar{\mu} \in R^{m}$ and $\bar{\vartheta} \in R^{q}$ (see, for example, [9]). We prove that $\bar{\lambda} \neq 0$. Suppose, contrary to the result, that $\bar{\lambda}=0$. Hence, as it follows from the necessary optimality conditions of F. John type (3.3), we have $(\bar{\lambda}, \bar{\mu}) \geq 0$. Since $\bar{\lambda}=0$, the above relation implies that $\bar{\mu} \neq 0$. Using $\bar{\lambda}=0$ together with the necessary optimality conditions of F. John type (3.1), we get

$$
0 \in \sum_{j=1}^{m} \bar{\mu}_{j} \partial g_{j}(\bar{x})+\sum_{t=1}^{q} \bar{\vartheta}_{t} \partial h_{t}(\bar{x})
$$


By (3.4), there exist $\zeta_{j} \in \partial g_{j}(\bar{x}), j \in J$ and $\varsigma_{t} \in \partial h_{t}(\bar{x}), t \in T$ such that

$$
0=\sum_{j=1}^{m} \bar{\mu}_{j} \zeta_{j}+\sum_{t=1}^{q} \bar{\vartheta}_{t} \varsigma_{t}
$$

Since $\bar{\mu} \neq 0$, we have that $A=\sum_{j=1}^{m} \bar{\mu}_{j}+\sum_{t \in T^{+}(\bar{x})} \bar{\vartheta}_{t}-\sum_{t \in T^{-}(\bar{x})} \bar{\vartheta}_{t}>0$. Let us denote

$$
\begin{gathered}
\widehat{\beta}_{j}=\frac{\bar{\mu}_{j}}{\sum_{j=1}^{m} \bar{\mu}_{j}+\sum_{t \in T^{+}(\bar{x})} \bar{\vartheta}_{t}-\sum_{t \in T^{-}(\bar{x})} \bar{\vartheta}_{t}}, j \in J(\bar{x}), \\
\widehat{\gamma}_{t}^{+}=\frac{\bar{\vartheta}_{t}}{\sum_{j=1}^{m} \bar{\mu}_{j}+\sum_{t \in T^{+}(\bar{x})} \bar{\vartheta}_{t}-\sum_{t \in T^{-}(\bar{x})} \bar{\vartheta}_{t}}, t \in T^{+}(\bar{x}), \\
\widehat{\gamma}_{t}^{-}=\frac{-\vartheta_{t}}{\sum_{j=1}^{m} \bar{\mu}_{j}+\sum_{t \in T^{+}(\bar{x})} \bar{\vartheta}_{t}-\sum_{t \in T^{-}(\bar{x})} \bar{\vartheta}_{t}}, t \in T^{-}(\bar{x}) .
\end{gathered}
$$

By (3.6)-(3.8), it follows that $\beta=\left(\widehat{\beta}_{1}, \ldots, \widehat{\beta}_{m}\right) \geq 0,0 \leqq \widehat{\beta}_{j} \leqq 1, j \in J(\bar{x})$, $0 \leqq \widehat{\gamma}_{t}^{+} \leqq 1, t \in T^{+}(\bar{x}), 0 \leqq \widehat{\gamma}_{t}^{-} \leqq 1, t \in T^{-}(\bar{x})$, and, moreover, $\sum_{j \in J(\bar{x})} \widehat{\beta}_{j}+\sum_{t \in T^{+}(\bar{x})} \widehat{\gamma}_{t}^{+}$ $+\sum_{t \in T^{-}(\bar{x})} \widehat{\gamma}_{t}^{-}=1$. By assumption, $g_{j}, j \in J(\bar{x})$, is $\left(\Phi, \rho_{g_{j}}\right)^{w}$-invex at $\bar{x}$ on $D, h_{t}$, $t \in T^{+}(\bar{x}):=\left\{t \in T: \bar{\vartheta}_{t}>0\right\}$, is locally Lipschitz $\left(\Phi, \rho_{h_{t}}^{+}\right)^{w}$-invex at $\bar{x}$ on $D,-h_{t}$, $t \in T^{-}(\bar{x}):=\left\{t \in T: \bar{\vartheta}_{t}<0\right\}$, is locally Lipschitz $\left(\Phi, \rho_{h_{t}}^{-}\right)^{w}$-invex at $\bar{x}$ on $D$. Further, as it follows from the assumption, there exists $\widetilde{x} \in D$ such that $g_{j}(\widetilde{x})<0$, $j \in J(\bar{x})$. Hence, by Definition 5, it follows that the following inequalities

$$
\begin{gathered}
g_{j}(\widetilde{x})-g_{j}(\bar{x}) \geqq \Phi\left(\widetilde{x}, \bar{x},\left(\zeta_{j}, \rho_{g_{j}}\right)\right), \quad j \in J(\bar{x}), \\
h_{t}(\widetilde{x})-h_{t}(\bar{x}) \geqq \Phi\left(\widetilde{x}, \bar{x},\left(\varsigma_{t}, \rho_{h_{t}}^{+}\right)\right), \quad t \in T^{+}(\bar{x}), \\
-h_{t}(\widetilde{x})+h_{t}(\bar{x}) \geqq \Phi\left(\widetilde{x}, \bar{x},\left(-\varsigma_{t}, \rho_{h_{t}}^{-}\right)\right), \quad t \in T^{-}(\bar{x}) .
\end{gathered}
$$

hold for each $\xi_{i} \in \partial f_{i}(\bar{x}), i \in I(\bar{x}), \zeta_{j} \in \partial g_{j}(\bar{x}), j \in J(\bar{x}), \varsigma_{t} \in \partial h_{t}(\bar{x}), t \in T^{+}(\bar{x}) \cup$ $T^{-}(\bar{x})$. Combining $g_{j}(\widetilde{x})<0, j \in J(\bar{x})$ and (3.9), we get

$$
\Phi\left(\widetilde{x}, \bar{x},\left(\zeta_{j}, \rho_{g_{j}}\right)\right)<0, \quad j \in J(\bar{x}) .
$$

By $\widetilde{x} \in D, \bar{x} \in D$, inequalities (3.10) and (3.11) yield, respectively

$$
\begin{gathered}
\Phi\left(\tilde{x}, \bar{x},\left(\varsigma_{t}, \rho_{h_{t}}^{+}\right)\right) \leqq 0, \quad t \in T^{+}(\bar{x}), \\
\Phi\left(\tilde{x}, \bar{x},\left(-\varsigma_{t}, \rho_{h_{t}}^{-}\right)\right) \leqq 0, \quad t \in T^{-}(\bar{x}) .
\end{gathered}
$$

By Definition 5 , we have that $\Phi(\widetilde{x}, \bar{x},(\cdot, \cdot))$ is a strictly quasi-convex function on $R^{n+1}$. Since (3.12)-(3.14) are satisfied, by Proposition 1, it follows that

$$
\Phi\left(\tilde{x}, \bar{x},\left(\sum_{j \in J(\bar{x})} \widehat{\beta}_{j} \zeta_{j}+\sum_{t \in T^{+}(\bar{x})} \widehat{\gamma}_{t}^{+} \varsigma_{t}+\sum_{t \in T^{-}(\bar{x})} \widehat{\gamma}_{t}^{-}\left(-\varsigma_{t}\right),\right.\right.
$$




$$
\left.\left.\sum_{j \in J(\bar{x})} \widehat{\beta}_{j} \rho_{g_{j}}+\sum_{t \in T^{+}(\bar{x})} \widehat{\gamma}_{t}^{+} \rho_{h_{t}}^{+}+\sum_{t \in T^{-}(\bar{x})} \widehat{\gamma}_{t}^{-} \rho_{h_{t}}^{-}\right)\right)<0 .
$$

Taking into account (3.6)-(3.8) and Lagrange multipliers equal to 0 in (3.15), we get

$$
\Phi\left(\tilde{x}, \bar{x}, \frac{1}{A}\left(\sum_{j=1}^{m} \bar{\mu}_{j} \zeta_{j}+\sum_{t=1}^{q} \bar{\vartheta}_{t} \varsigma_{t}, \sum_{j=1}^{m} \bar{\mu}_{j} \rho_{g_{j}}+\sum_{t \in T^{+}(\bar{x})} \bar{\vartheta}_{t} \rho_{h_{t}}^{+}-\sum_{t \in T^{-}(\bar{x})} \bar{\vartheta}_{t} \rho_{h_{t}}^{-}\right)\right)<0 .
$$

By the necessary optimality condition of F. John type (3.1), it follows that

$$
\Phi\left(\widetilde{x}, \bar{x}, \frac{1}{A}\left(0, \sum_{j=1}^{m} \bar{\mu}_{j} \rho_{g_{j}}+\sum_{t \in T^{+}(\bar{x})} \bar{\vartheta}_{t} \rho_{h_{t}}^{+}-\sum_{t \in T^{-}(\bar{x})} \bar{\vartheta}_{t} \rho_{h_{t}}^{-}\right)\right)<0 .
$$

By assumption, $\sum_{j=1}^{m} \bar{\mu}_{j} \rho_{g_{j}}+\sum_{t \in T^{+}(\bar{x})} \bar{\vartheta}_{t} \rho_{h_{t}}^{+}-\sum_{t \in T^{-}(\bar{x})} \bar{\vartheta}_{t} \rho_{h_{t}}^{-} \geqq 0$. Thus, by Definition 5 , the following inequality

$$
\Phi\left(\widetilde{x}, \bar{x}, \frac{1}{A}\left(0, \sum_{j=1}^{m} \bar{\mu}_{j} \rho_{g_{j}}+\sum_{t \in T^{+}(\bar{x})} \bar{\vartheta}_{t} \rho_{h_{t}}^{+}-\sum_{t \in T^{-}(\bar{x})} \bar{\vartheta}_{t} \rho_{h_{t}}^{-}\right)\right) \geqq 0
$$

holds, contradicts (3.17). This means that $\bar{\lambda} \neq 0$. If $\sum_{i=1}^{k} \bar{\lambda}_{i} \neq 1$, then it is sufficient to normalize the Lagrange multipliers $\bar{\lambda}_{i}, i \in I$. This completes the proof of this theorem.

Remark 2. Theorem 2 can also be proved if hypotheses that each function $h_{t}$, $t \in T^{+}(\bar{x}):=\left\{t \in T: \bar{\vartheta}_{t}>0\right\}$, is locally Lipschitz $\left(\Phi, \rho_{h_{t}}\right)^{w}$-invex at $\bar{x}$ on $D$ and each function $-h_{t}, t \in T^{-}(\bar{x}):=\left\{t \in T: \bar{\vartheta}_{t}<0\right\}$, is locally Lipschitz $\left(\Phi, \rho_{h_{t}}\right)^{w}$-invex at $\bar{x}$ on $D$ are replaced by, in general, a weaker hypothesis that $\sum_{t=1}^{q} \bar{\vartheta}_{t} h_{t}$ is locally Lipschitz $\left(\Phi, \rho_{h}\right)^{w}$-invex at $\bar{x}$ on $D$.

Definition 8. The point $(\bar{x}, \bar{\lambda}, \bar{\mu}, \bar{\vartheta}) \in D \times R^{k} \times R^{m} \times R^{q}$ is said to be a KarushKuhn-Tucker point of the considered vector optimization problem (VP) if the necessary optimality conditions (3.1)-(3.2) and, in place of (3.3), the conditions $\bar{\lambda} \geq 0$, $\sum_{i=1}^{k} \bar{\lambda}_{i}=1, \bar{\mu} \geqq 0$ are satisfied at $\bar{x}$ with Lagrange multipliers $\bar{\lambda}, \bar{\mu}$ and $\bar{\vartheta}$.

Now, we prove the sufficient optimality conditions for weak efficiency of a feasible solution in the considered nonsmooth multiobjective programming problem (VP) under nonsmooth $(\Phi, \rho)^{w}$-invexity.

Theorem 3. Let $(\bar{x}, \bar{\lambda}, \bar{\mu}, \bar{\vartheta}) \in D \times R^{k} \times R^{m} \times R^{q}$ be a Karush-Kuhn-Tucker point of the considered nonsmooth multiobjective programming problem (VP). Further, assume that $f_{i}, i \in I$, is locally Lipschitz $\left(\Phi, \rho_{f_{i}}\right)^{w}$-invex at $\bar{x}$ on $D, g_{j}, j \in J(\bar{x})$, is locally Lipschitz $\left(\Phi, \rho_{g_{j}}\right)^{w}$-invex at $\bar{x}$ on $D, h_{t}, t \in T^{+}(\bar{x}):=\{t \in T: \bar{\vartheta}>0\}$, is locally Lipschitz $\left(\Phi, \rho_{h_{t}}^{+}\right)^{w}$-invex at $\bar{x}$ on $D,-h_{t}, t \in T^{-}(\bar{x}):=\{t \in T: \bar{\vartheta}<0\}$, is locally 
Lipschitz $\left(\Phi, \rho_{h_{t}}^{-}\right)^{w}$-invex at $\bar{x}$ on D. If $\sum_{i=1}^{k} \bar{\lambda}_{i} \rho_{f_{i}}+\sum_{j \in J(\bar{x})} \bar{\mu}_{j} \rho_{g_{j}}+\sum_{t \in T^{+}(\bar{x})} \bar{\vartheta}_{t} \rho_{h_{t}}^{+}-$ $\sum_{t \in T^{-}(\bar{x})} \bar{\vartheta}_{t} \rho_{h_{t}}^{-} \geqq 0$, then $\bar{x}$ is a weak Pareto optimal solution of the problem (VP).

Proof. Suppose, contrary to the result, that $\bar{x}$ is not a weak Pareto optimal solution of the problem (VP). Then, by Definition 6, there exists a feasible solution $\widetilde{x}$ such that

$$
f(\widetilde{x})<f(\bar{x}) .
$$

By assumption, $(\bar{x}, \bar{\lambda}, \bar{\mu}, \bar{\vartheta}) \in D \times R^{k} \times R^{m} \times R^{q}$ is a Karush-Kuhn-Tucker point of the considered nonsmooth multiobjective programming problem (VP). Then, by Definition 8, the necessary optimality conditions (3.1)-(3.2) hold with $\bar{\lambda} \geq 0, \sum_{i=1}^{k} \bar{\lambda}_{i}=1$. By (3.1), there exist $\xi_{i} \in \partial f_{i}(\bar{x}), i \in I, \zeta_{j} \in \partial g_{j}(\bar{x}), j \in J, \varsigma_{t} \in \partial h_{t}(\bar{x}), t \in T$, such that

$$
\sum_{i=1}^{k} \bar{\lambda}_{i} \xi_{i}+\sum_{j \in J(\bar{x})} \bar{\mu}_{j} \zeta_{j}+\sum_{t \in T^{+}(\bar{x})} \bar{\vartheta}_{t} \varsigma_{t}+\sum_{t \in T^{-}(\bar{x})} \bar{\vartheta}_{t} \varsigma_{t}=0
$$

As it follows from Definition 5, the following inequality $\Phi(\widetilde{x}, \bar{x},(0, a)) \geqq 0$ holds for each $a \geqq 0$. Hence, by (3.19), hypothesis $\sum_{i \in I(\bar{x})} \bar{\lambda}_{i} \rho_{f_{i}}+\sum_{j \in J(\bar{x})} \bar{\mu}_{j} \rho_{g_{j}}+\sum_{t \in T^{+}(\bar{x})} \bar{\vartheta}_{t} \rho_{h_{t}}^{+}-$ $\sum_{t \in T^{-}(\bar{x})} \bar{\vartheta}_{t} \rho_{h_{t}}^{-} \geqq 0$ implies

$$
\begin{aligned}
& \Phi\left(\tilde{x}, \bar{x}, \frac{1}{\bar{A}}\left(\sum_{i=1}^{k} \bar{\lambda}_{i} \xi_{i}+\sum_{j \in J(\bar{x})} \bar{\mu}_{j} \zeta_{j}+\sum_{t \in T^{+}(\bar{x})} \bar{\vartheta}_{t} \varsigma_{t}+\sum_{t \in T^{-}(\bar{x})} \bar{\vartheta}_{t} \varsigma_{t},\right.\right. \\
& \left.\left.\sum_{i=1}^{k} \bar{\lambda}_{i} \rho_{f_{i}}+\sum_{j \in J(\bar{x})} \bar{\mu}_{j} \rho_{g_{j}}+\sum_{t \in T^{+}(\bar{x})} \bar{\vartheta}_{t} \rho_{h_{t}}^{+}-\sum_{t \in T^{-}(\bar{x})} \bar{\vartheta}_{t} \rho_{h_{t}}^{-}\right)\right) \geqq 0,
\end{aligned}
$$

where

$$
\bar{A}=\sum_{i=1}^{k} \bar{\lambda}_{i}+\sum_{j=1}^{m} \bar{\mu}_{j}+\sum_{t \in T^{+}(\bar{x})} \bar{\vartheta}_{t}-\sum_{t \in T^{-}(\bar{x})} \bar{\vartheta}_{t}>0
$$

Let us denote

$$
\begin{gathered}
\bar{\alpha}_{i}=\frac{\bar{\lambda}_{i}}{\bar{A}}, i \in I(\bar{x}), \bar{\beta}_{j}=\frac{\bar{\mu}_{j}}{\bar{A}}, j \in J(\bar{x}), \\
\bar{\gamma}_{t}^{+}=\frac{\bar{\vartheta}_{t}}{\bar{A}}, t \in T^{+}(\bar{x}), \bar{\gamma}_{t}^{-}=\frac{-\bar{\vartheta}_{t}}{\bar{A}}, t \in T^{-}(\bar{x}) .
\end{gathered}
$$

Then, by $\bar{\lambda} \geq 0, \sum_{i=1}^{k} \bar{\lambda}_{i}=1$, it follows that $\bar{\alpha}:=\left(\bar{\alpha}_{1}, \ldots, \bar{\alpha}_{k}\right) \geq 0,0 \leqq \bar{\alpha}_{i} \leqq 1, i \in I$, $0<\bar{\alpha}_{i} \leqq 1$ for at least one $i \in I, \bar{\beta}=\left(\bar{\beta}_{1}, \ldots, \bar{\beta}_{m}\right) \geqq 0,0 \leqq \bar{\beta}_{j} \leqq 1, j \in J, 0 \leqq \bar{\gamma}_{t}^{+} \leqq 1$, $t \in T^{+}(\bar{x}), 0 \leqq \bar{\gamma}_{t}^{-} \leqq 1, t \in T^{-}(\bar{x})$, and, moreover,

$$
\sum_{i=1}^{k} \bar{\alpha}_{i}+\sum_{j \in J(\bar{x})} \bar{\beta}_{j}+\sum_{t \in T^{+}(\bar{x})} \bar{\gamma}_{t}^{+}+\sum_{t \in T^{-}(\bar{x})} \bar{\gamma}_{t}^{-}=1 .
$$


Since $f_{i}, i \in I$, is locally Lipschitz $\left(\Phi, \rho_{f_{i}}\right)^{w}$-invex at $\bar{x}$ on $D, g_{j}, j \in J(\bar{x})$, is $\left(\Phi, \rho_{g_{i}}\right)^{w}$ invex at $\bar{x}$ on $D, h_{t}, t \in T^{+}(\bar{x}):=\{t \in T: \bar{\vartheta}>0\}$, is locally Lipschitz $\left(\Phi, \rho_{h_{t}}^{+}\right)^{w}-$ invex at $\bar{x}$ on $D,-h_{t}, t \in T^{-}(\bar{x}):=\{t \in T: \bar{\vartheta}<0\}$, is locally Lipschitz $\left(\Phi, \rho_{h_{t}}^{-}\right)^{w}-$ invex at $\bar{x}$ on $D$, by Definition 5 , it follows that the following inequalities

$$
\begin{gathered}
f_{i}(\widetilde{x})-f_{i}(\bar{x}) \geqq \Phi\left(\widetilde{x}, \bar{x},\left(\xi_{i}, \rho_{f_{i}}\right)\right), \quad i \in I, \\
g_{j}(\widetilde{x})-g_{j}(\bar{x}) \geqq \Phi\left(\widetilde{x}, \bar{x},\left(\zeta_{j}, \rho_{g_{j}}\right)\right), \quad j \in J(\bar{x}), \\
h_{t}(\widetilde{x})-h_{t}(\bar{x}) \geqq \Phi\left(\widetilde{x}, \bar{x},\left(\varsigma_{t}, \rho_{h_{t}}^{+}\right)\right), \quad t \in T^{+}(\bar{x}), \\
-h_{t}(\widetilde{x})+h_{t}(\bar{x}) \geqq \Phi\left(\widetilde{x}, \bar{x},\left(-\varsigma_{t}, \rho_{h_{t}}^{-}\right)\right), \quad t \in T^{-}(\bar{x})
\end{gathered}
$$

hold for each $\xi_{i} \in \partial f_{i}(\bar{x}), i \in I, \zeta_{j} \in \partial g_{j}(\bar{x}), j \in J(\bar{x}), \varsigma_{t} \in \partial h_{t}(\bar{x}), t \in T^{+}(\bar{x}) \cup T^{-}(\bar{x})$, respectively. By (3.18), inequality (3.25) implies

$$
\Phi\left(\widetilde{x}, \bar{x},\left(\xi_{i}, \rho_{f_{i}}\right)\right)<0, \quad i \in I .
$$

By $\widetilde{x} \in D, \bar{x} \in D$, inequalities (3.26)-(3.28) yield, respectively,

$$
\begin{gathered}
\Phi\left(\widetilde{x}, \bar{x},\left(\zeta_{j}, \rho_{g_{j}}\right)\right) \leqq 0, \quad j \in J(\bar{x}), \\
\Phi\left(\widetilde{x}, \bar{x},\left(\varsigma_{t}, \rho_{h_{t}}^{+}\right)\right) \leqq 0, \quad t \in T^{+}(\bar{x}), \\
\Phi\left(\tilde{x}, \bar{x},\left(-\varsigma_{t}, \rho_{h_{t}}^{-}\right)\right) \leqq 0, \quad t \in T^{-}(\bar{x}) .
\end{gathered}
$$

As it follows from Definition $5, \Phi(\widetilde{x}, \bar{x}, \cdot)$ is a strictly quasi-convex function on $R^{n+1}$. Since (3.24) is satisfied, by inequalities (3.29)-(3.32), Proposition 1 implies

$$
\begin{aligned}
& \Phi(\widetilde{x}, \bar{x},\left(\sum_{i \in I(\bar{x})} \bar{\alpha}_{i} \xi_{i}+\sum_{j \in J(\bar{x})} \bar{\beta}_{j} \zeta_{j}+\sum_{t \in T^{+}(\bar{x})} \bar{\gamma}_{t}^{+} \varsigma_{t}+\sum_{t \in T^{-}(\bar{x})} \bar{\gamma}_{t}^{-}\left(-\varsigma_{t}\right),\right. \\
&\left.\left.\sum_{i \in I(\bar{x})} \bar{\alpha}_{i} \rho_{f_{i}}+\sum_{j \in J(\bar{x})} \bar{\beta}_{j} \rho_{g_{j}}+\sum_{t \in T^{+}(\bar{x})} \bar{\gamma}_{t}^{+} \rho_{h_{t}}^{+}+\sum_{t \in T^{-}(\bar{x})} \bar{\gamma}_{t}^{-} \rho_{h_{t}}^{-}\right)\right)<0 .
\end{aligned}
$$

Taking into account (3.22)-(3.23) in (3.33), we obtain that the following inequality

$$
\begin{gathered}
\Phi\left(\tilde{x}, \bar{x}, \frac{1}{\bar{A}}\left(\sum_{i=1}^{k} \bar{\lambda}_{i} \xi_{i}+\sum_{j \in J(\bar{x})} \bar{\mu}_{j} \zeta_{j}+\sum_{t \in T^{+}(\bar{x})} \bar{\vartheta}_{t} \varsigma_{t}+\sum_{t \in T^{-}(\bar{x})} \bar{\vartheta}_{t} \varsigma_{t},\right.\right. \\
\left.\left.\sum_{i \in I(\bar{x})} \bar{\lambda}_{i} \rho_{f_{i}}+\sum_{j \in J(\bar{x})} \bar{\mu}_{j} \rho_{g_{j}}+\sum_{t \in T^{+}(\bar{x})} \bar{\vartheta}_{t} \rho_{h_{t}}^{+}-\sum_{t \in T^{-}(\bar{x})} \bar{\vartheta}_{t} \rho_{h_{t}}^{-}\right)\right)<0
\end{gathered}
$$

holds, contradicting (3.20). This means that $\bar{x}$ is a weakly efficient solution of (VP) and completes the proof of this theorem. 
In order to prove the sufficient optimality conditions for a Pareto optimal solution of the nonsmooth multiobjective programming problem (VP) with nonsmooth $(\Phi, \rho)$ invex functions, some stronger hypotheses should be assumed.

Theorem 4. Let $(\bar{x}, \bar{\lambda}, \bar{\mu}, \bar{\vartheta}) \in D \times R^{k} \times R^{m} \times R^{q}$ be a Karush-Kuhn-Tucker point of the considered nonsmooth multiobjective programming problem (VP). Further, assume that any one of the following hypotheses is satisfied:

i) the objective function $f_{i}, i \in I$, is locally Lipschitz strictly $\left(\Phi, \rho_{f_{i}}\right)^{w}$-invex at $\bar{x}$ on $D$, the constraint function $g_{j}, j \in J(\bar{x})$, is locally Lipschitz $\left(\Phi, \rho_{g_{j}}\right)^{w}$-invex at $\bar{x}$ on $D, h_{t}, t \in T^{+}(\bar{x}):=\left\{t \in T: \bar{\vartheta}_{t}>0\right\}$, is locally Lipschitz $\left(\Phi, \rho_{h_{t}}^{+}\right)^{w}-$ invex at $\bar{x}$ on $D,-h_{t}, t \in T^{-}(\bar{x}):=\left\{t \in T: \bar{\vartheta}_{t}<0\right\}$, is locally Lipschitz $\left(\Phi, \rho_{h_{t}}^{-}\right)^{w}$-invex at $\bar{x}$ on $D$ and, moreover, $\sum_{i=1}^{k} \bar{\lambda}_{i} \rho_{f_{i}}+\sum_{j \in J(\bar{x})} \bar{\mu}_{j} \rho_{g_{j}}+$ $\sum_{t \in T^{+}(\bar{x})} \bar{\vartheta}_{t} \rho_{h_{t}}^{+}-\sum_{t \in T^{-}(\bar{x})} \bar{\vartheta}_{t} \rho_{h_{t}}^{-} \geqq 0$,

ii) the Lagrange multipliers $\bar{\lambda}_{i}>0, i \in I$, the objective function $f_{i}, i \in I$, is locally Lipschitz $\left(\Phi, \rho_{f_{i}}\right)^{w}$-invex at $\bar{x}$ on $D$, the constraint function $g_{j}, j \in J(\bar{x})$, is locally Lipschitz $\left(\Phi, \rho_{g_{j}}\right)$-invex at $\bar{x}$ on $D, h_{t}, t \in T^{+}(\bar{x}):=\left\{t \in T: \bar{\vartheta}_{t}>0\right\}$, is locally Lipschitz $\left(\Phi, \rho_{h_{t}}^{+}\right)^{w}$-invex at $\bar{x}$ on $D, \quad-h_{t}, t \in T^{-}(\bar{x}):=$ $\left\{t \in T: \bar{\vartheta}_{t}<0\right\}$, is locally Lipschitz $\left(\Phi, \rho_{h_{t}}^{-}\right)^{w}$-invex at $\bar{x}$ on $D$ and $\sum_{i=1}^{k} \bar{\lambda}_{i} \rho_{f_{i}}+\sum_{j \in J(\bar{x})} \bar{\mu}_{j} \rho_{g_{j}}+\sum_{t \in T^{+}(\bar{x})} \bar{\vartheta}_{t} \rho_{h_{t}}^{+}-\sum_{t \in T^{-}(\bar{x})} \bar{\vartheta}_{t} \rho_{h_{t}}^{-} \geqq 0$.

Then $\bar{x}$ is an efficient solution of the problem (VP).

Proof. The proof of this theorem is similar to the proof of Theorem 3.

In order to illustrate the sufficient optimality results established in this section, we consider the following example of a nondifferentiable multiobjective programming problem with $(\Phi, \rho)^{w}$-invex functions, not necessarily, with respect to the same $\rho$.

Example 1. Consider the following nondifferentiable multiobjective programming problem defined as follows

$$
\begin{gathered}
f(x)=\left(\arctan \left(\left|x_{1}\right|\right)+\arctan \left(\left|x_{2}\right|\right), x_{1}^{2}+x_{2}^{2}+\arctan \left(\left|x_{1} x_{2}\right|\right)\right) \rightarrow V-\min \\
g(x)=\left|x_{1}\right|+\left|x_{2}\right|-2 \arctan \left(\left|x_{1} x_{2}\right|\right) \leqq 0, \quad(V P 1) \\
X=R^{2} .
\end{gathered}
$$

It is not difficult to see that $D=\left\{\left(x_{1}, x_{2}\right) \in R^{2}:\left|x_{1}\right|+\left|x_{2}\right|-2 \arctan \left(\left|x_{1} x_{2}\right|\right) \leqq 0\right\}$ and $\bar{x}=(0,0)$ is such a feasible point at which the Generalized Karush-Kuhn-Tucker necessary optimality conditions are satisfied. It can be established, by Definition 5, that the objective function $f_{i}, i=1,2$, is locally Lipschitz strictly $\left(\Phi, \rho_{f_{i}}\right)^{w}$-invex at $\bar{x}$ on $D$ and the constraint function $g$ is $\left(\Phi, \rho_{g}\right)^{w}$-invex at $\bar{x}$ on $D$, where

$$
\Phi(x, \bar{x},(\varsigma, \rho))=\arctan \left(\varsigma_{1}\left|x_{1}\right|\right)+\arctan \left(\varsigma_{2}\left|x_{2}\right|\right)+\arctan (\rho)\left(\arctan \left|x_{1} x_{2}\right|-\arctan \left|\bar{x}_{1} \bar{x}_{2}\right|\right)
$$




$$
\rho_{f_{1}}=0, \rho_{f_{2}}=\tan (1), \rho_{g}=\tan (-2),
$$

and $\varsigma \in \partial k(\bar{x})$, where $k$ denotes $f_{1}$ or $f_{2}$ or $g$, respectively, and $\rho$ is equal to $\rho_{f_{1}}, \rho_{f_{2}}$ or $\rho_{g}$, respectively.

Since all hypotheses of Theorem 4 are satisfied, $\bar{x}$ is an efficient solution of the considered nonsmooth multiobjective programming problem. Note that we are not in a position to prove efficiency of $\bar{x}$ in the considered nonconvex nonsmooth multiobjective programming problem (VP1) under other generalized convexity notions existing in the literature, that is, invexity [14,16], $b$-invexity [17], $F$-convexity [10], $r$-invexity [3], $V$-invexity [13], $G$-invexity [4], $V$ - $r$-invexity [5], univexity [18]. This follows from the fact that not every stationary point of the functions constituting problem (VP1) is a global minimum of such a function. Whereas one of the main property of the concepts generalized convexity notions mentioned above is that a stationary point of every function belonging to the aforesaid classes of generalized convex functions is its global minimizer. Further, we can't use also the sufficient optimality conditions under nondifferentiable $(\Phi, \rho)$-invexity since the functional $\Phi(x, \bar{x}, \cdot)$ is not convex for all $x \in D$ as it follows from the definition of this concept of generalized convexity (see [6]). As it follows even from this example, the introduced concept of nondifferentiable $(\Phi, \rho)^{w}$-invexity is useful to prove the sufficiency of Generalized Karush-Kuhn-Tucker necessary optimality conditions for a larger class of nonconvex nondifferentiable vector optimization problems in comparison to other generalized convexity notions, earlier defined in the literature.

\section{MOND-Weir DUALity}

In this section, for the considered nonsmooth multiobjective programming problem (VP), we define a vector dual problem in the Mond-Weir sense. Then, we prove several duality results between the primal multiobjective programming problem and its Mond-Weir dual problem under $(\Phi, \rho)^{w}$-invexity hypotheses.

Now, for the considered nonsmooth multiobjective programming problem (VP), we state the following vector Mond-Weir dual problems as follows:

$$
\begin{gathered}
f(y) \rightarrow V \text {-max } \\
\text { s.t. } 0 \in \sum_{i=1}^{k} \lambda_{i} \partial f_{i}(y)+\sum_{j=1}^{m} \mu_{j} \partial g_{j}(y)+\sum_{t=1}^{q} \vartheta_{t} \partial h_{t}(y), \\
\sum_{j=1}^{m} \mu_{j} g_{j}(y) \geqq 0, \quad \sum_{t=1}^{q} \vartheta_{t} h_{t}(y) \geqq 0, \\
\lambda \in R^{k}, \lambda \geq 0, \sum_{i=1}^{k} \lambda_{i}=1, \mu \in R^{m}, \mu \geqq 0, \vartheta \in R^{q} .
\end{gathered}
$$

We denote by $\Omega$ the set of all feasible solutions in the vector Mond-Weir dual problem (VD) and, moreover, let $Y$ be the projection of the set $\Omega$ on $X$, that is, $Y=\{y \in X:(y, \lambda, \mu, \vartheta) \in \Omega\}$. 
Theorem 5 (Weak duality). Let $x$ and $(y, \lambda, \mu, \vartheta)$ be any feasible solutions for the problems (VP) and (VD), respectively. Further, assume that $f_{i}, i \in I$, is locally Lipschitz $\left(\Phi, \rho_{f}\right)^{w}$-invex at $y$ on $D \cup Y, \sum_{j=1}^{m} \mu_{j} g_{j}$ is locally Lipschitz $\left(\Phi, \rho_{g}\right)^{w}$ invex at $y$ on $D \cup Y, \sum_{t=1}^{q} \vartheta_{t} h_{t}$ is locally Lipschitz $\left(\Phi, \rho_{h}\right)^{w}$-invex at $y$ on $D \cup Y$. If $\sum_{i=1}^{k} \lambda_{i} \rho_{f_{i}}+\rho_{g}+\rho_{h} \geqq 0$, then $f(x) \nless f(y)$.

Proof. We proceed by contradiction. Suppose, contrary to the result, that there exist $x \in D$ and $(y, \lambda, \mu, \vartheta) \in \Omega$ such that

$$
f(x)<f(y) .
$$

By assumption, $f_{i}, i \in I$, is locally Lipschitz $\left(\Phi, \rho_{f}\right)^{w}$-invex at $y$ on $D \cup Y$. Hence, by Definition 5, the following inequalities

$$
f_{i}(z)-f_{i}(y) \geqq \Phi\left(z, y,\left(\xi_{i}, \rho_{f_{i}}\right)\right), i \in I
$$

hold for all $z \in D \cup Y$ and for each $\xi_{i} \in \partial f_{i}(y)$. Therefore, they are also satisfied for $z=x \in D$. Thus, inequalities (4.2) yield

$$
f_{i}(x)-f_{i}(y) \geqq \Phi\left(x, y,\left(\xi_{i}, \rho_{f_{i}}\right)\right), i \in I .
$$

Combining (4.1) and (4.3), we have

$$
\Phi\left(x, y,\left(\xi_{i}, \rho_{f_{i}}\right)\right)<0, i \in I .
$$

By assumptions, $\sum_{j=1}^{m} \mu_{j} g_{j}$ is locally Lipschitz $\left(\Phi, \rho_{g}\right)^{w}$-invex at $y$ on $D \cup Y, \sum_{t=1}^{q} \vartheta_{t} h_{t}$ is locally Lipschitz $\left(\Phi, \rho_{h}\right)^{w}$-invex at $y$ on $D \cup Y$. Hence, by Definition 5, the following inequalities

$$
\begin{gathered}
\sum_{j=1}^{m} \mu_{j} g_{j}(x)-\sum_{j=1}^{m} \mu_{j} g_{j}(y) \geqq \Phi\left(x, y,\left(\sum_{j=1}^{m} \mu_{j} \zeta_{j}, \rho_{g}\right)\right), \\
\sum_{t=1}^{q} \vartheta_{t} h_{t}(x)-\sum_{t=1}^{q} \vartheta_{t} h_{t}(y) \geqq \Phi\left(x, y,\left(\sum_{t=1}^{q} \vartheta_{t} \varsigma_{t}, \rho_{h}\right)\right)
\end{gathered}
$$

hold for each $\zeta_{j} \in \partial g_{j}(y), j \in J$ and $\zeta_{t} \in \partial h_{t}(y), t \in T$, respectively. By $x \in D$ and $(y, \lambda, \mu, \vartheta) \in \Omega,(4.5)$ and (4.6) yield, respectively,

$$
\begin{gathered}
\Phi\left(x, y,\left(\sum_{j=1}^{m} \mu_{j} \zeta_{j}, \rho_{g}\right)\right) \leqq 0, \\
\Phi\left(x, y,\left(\sum_{t=1}^{q} \vartheta_{t} \varsigma_{t}, \rho_{h}\right)\right) \leqq 0 .
\end{gathered}
$$

By Definition $5, \Phi(x, y, \cdot)$ is strictly quasi-convex on $R^{n+1}$. Then, by Proposition 1 , inequalities (4.4), (4.7) and (4.8) imply

$$
\Phi\left(x, y,\left(\sum_{i=1}^{k} \frac{\lambda_{i}}{3} \xi_{i}+\frac{1}{3} \sum_{j=1}^{m} \mu_{j} \zeta_{j}+\frac{1}{3} \sum_{t=1}^{q} \vartheta_{t} \varsigma_{t},\right.\right.
$$




$$
\left.\left.\sum_{i=1}^{k} \frac{\lambda_{i}}{3} \rho_{f_{i}}+\frac{1}{3} \rho_{g}+\frac{1}{3} \rho_{h}\right)\right)<0 .
$$

Thus, (4.9) gives

$$
\Phi\left(x, y, \frac{1}{3}\left(\sum_{i=1}^{k} \lambda_{i} \xi_{i}+\sum_{j=1}^{m} \mu_{j} \zeta_{j}+\sum_{t=1}^{q} \vartheta_{t} \varsigma_{t}, \sum_{i=1}^{k} \lambda_{i} \rho_{f_{i}}+\rho_{g}+\rho_{h}\right)\right)<0 .
$$

Using $(y, \lambda, \mu, \vartheta) \in \Omega$ again, the first constraint of dual problem (VD) gives

$$
\Phi\left(x, y, \frac{1}{3}\left(0, \sum_{i=1}^{k} \lambda_{i} \rho_{f_{i}}+\rho_{g}+\rho_{h}\right)\right)<0 .
$$

By Definition 5, it follows that $\Phi(x, y,(0, a)) \geqq 0$ for any $a \in R_{+}$. Therefore, hypothesis $\sum_{i=1}^{k} \lambda_{i} \rho_{f_{i}}+\rho_{g}+\rho_{h_{t}} \geqq 0$ implies that the following inequality

$$
\Phi\left(x, y, \frac{1}{3}\left(0, \sum_{i=1}^{k} \lambda_{i} \rho_{f_{i}}+\rho_{g}+\rho_{h}\right)\right) \geqq 0
$$

holds, contradicting (4.11). Hence, the proof of this theorem is completed.

If a stronger $\left(\Phi, \rho_{f}\right)^{w}$-invexity assumption is imposed on the objective functions constituting considered vector optimization problems, then the following stronger result can be established.

Theorem 6 (Weak duality). Let $x$ and $(y, \lambda, \mu, \vartheta)$ be feasible solutions for the problems $(V P)$ and $(V D)$, respectively. Further, assume that $f_{i}, i \in I$, is locally Lipschitz strictly $\left(\Phi, \rho_{f}\right)^{w}$-invex at y on $D \cup Y, \sum_{j=1}^{m} \mu_{j} g_{j}$ is locally Lipschitz $\left(\Phi, \rho_{g}\right)^{w}$ invex at $y$ on $D \cup Y, \sum_{t=1}^{q} \vartheta_{t} h_{t}$ is locally Lipschitz $\left(\Phi, \rho_{h}\right)^{w}$-invex at $y$ on $D \cup Y$. If $\sum_{i=1}^{k} \lambda_{i} \rho_{f_{i}}+\rho_{g}+\rho_{h} \geqq 0$, then $f(x) \not \leq f(y)$.

Theorem 7 (Strong duality). Let $\bar{x}$ be a weak Pareto solution (a Pareto solution) of the primal multiobjective programming (VP) and all hypotheses of Theorem 2 be satisfied at $\bar{x}$. Then there exist $\bar{\lambda} \in R^{k}, \bar{\mu} \in R^{m}$ and $\bar{\vartheta} \in R^{q}$ such that $(\bar{x}, \bar{\lambda}, \bar{\mu}, \bar{\vartheta})$ is feasible in (VD) and the objective functions of $(V P)$ and $(V D)$ are equal at these points. Further, if all hypotheses of the weak duality theorem (Theorem 5) are satisfied, then $(\bar{x}, \bar{\lambda}, \bar{\mu}, \bar{\vartheta})$ is a weakly efficient solution of a maximum type in (VD). If $\bar{\lambda}>0$ and all hypotheses of the weak duality theorem (Theorem 6) are satisfied, then $(\bar{x}, \bar{\lambda}, \bar{\mu}, \bar{\vartheta})$ is an efficient solution of a maximum type for the vector Mond-Weir dual problem (VD).

Proof. By assumption, $\bar{x} \in D$ is a weak Pareto optimal solution (a Pareto solution) of the problem (VP) and the constraint qualification is satisfied at $\bar{x}$. Then there exist the Lagrange multipliers $\bar{\lambda} \in R^{k}, \bar{\mu} \in R^{m}$ and $\bar{\vartheta} \in R^{q}$ such that the KarushKuhn-Tucker necessary optimality conditions (3.3)-(3.5) are satisfied at $\bar{x}$. Thus, the 
feasibility of $(\bar{x}, \bar{\lambda}, \bar{\mu}, \bar{\vartheta})$ in (VD) follows from these necessary optimality conditions and, moreover, $\bar{x} \in D$. Therefore, the objective functions of the problems (VP) and (VD) are equal at $\bar{x}$ and $(\bar{x}, \bar{\lambda}, \bar{\mu}, \bar{\vartheta})$. Hence, weakly efficiency of a maximum type of $(\bar{x}, \bar{\lambda}, \bar{\mu}, \bar{\vartheta})$ for (VD) follows directly from weak duality (Theorem 5), whereas efficiency of a maximum type of $(\bar{x}, \bar{\lambda}, \bar{\mu}, \bar{\vartheta})$ follows from Theorem 6 .

Theorem 8 (Converse duality). Let $(\bar{y}, \bar{\lambda}, \bar{\mu}, \bar{\vartheta})$ be an efficient solution of a maximum type (a weakly efficient solution of a maximum type) for the vector mixed dual problem (VD) such that $\bar{y} \in D$. Further, assume that $f_{i}, i \in I$, is (locally Lipschitz $\left(\Phi, \rho_{f}\right)^{w}$-invex) locally Lipschitz strictly $\left(\Phi, \rho_{f}\right)^{w}$-invex at $\bar{y}$ on $D \cup Y, \sum_{j=1}^{m} \bar{\mu}_{j} g_{j}$ is locally Lipschitz $\left(\Phi, \rho_{g}\right)^{w}$-invex at $\bar{y}$ on $D \cup Y, \sum_{t=1}^{q} \bar{\vartheta}_{t} h_{t}$ is locally Lipschitz $\left(\Phi, \rho_{h}\right)^{w}$ invex at $\bar{y}$ on $D \cup Y$. If $\sum_{i=1}^{k} \bar{\lambda}_{i} \rho_{f_{i}}+\rho_{g}+\rho_{h} \geqq 0$. Then $\bar{y}$ is an efficient solution (a weakly efficient solution) of the considered multiobjective programming problem $(V P)$.

Proof. The proof of the theorem follows directly from weak duality (Theorem 5 or Theorem 6, respectively).

\section{CONCLUSions}

In the paper, a new class of nonconvex nonsmooth multiobjective programming problems is considered in which every component of functions involved is locally Lipschitz $(\Phi, \rho)^{w}$-invex. Hence, the sufficient optimality conditions for weak efficiency and efficiency and duality results in the sense of Mond-Weir have been established for the considered nonconvex nonsmooth multiobjective programming problem under the concept of nondifferentiable $(\Phi, \rho)^{w}$-invexity introduced in the paper. Note that the definition of nondifferentiable $(\Phi, \rho)^{w}$-invexity unifies many generalized convex notions earlier introduced in the literature (see Remark 1). In order to illustrate the results established in the paper, some example of a nonconvex nonsmooth multiobjective programming problem with nondifferentiable $(\Phi, \rho)^{w}$-invex functions has been presented. It is interesting that not all functions constituting the considered nonsmooth vector optimization problem have the fundamental property of the most classes of generalized convex functions, namely that a stationary point of such a function is also its global minimum. Thus, we have also shown that many generalized convexity notions existing in the literature (that is, invexity [14], $b$-invexity [17], $F$-convexity [10], univexity [18], $r$-invexity [3], $V$-invexity [19], $V$ - $r$-invexity [5], $G$-invexity [4]) may fail in proving the sufficiency of the Karush-Kuhn-Tucker necessary optimality conditions and Mond-Weir duality results for the considered nonconvex nonsmooth vector optimization problem. Thus, the concept of nondifferentiable $(\Phi, \rho)^{w}$-invexity extend the class of nonconvex nonsmooth multiobjective 
programming problems for which it is possible to prove the sufficiency of the Generalized Karush-Kuhn-Tucker necessary optimality conditions and several duality theorems in the sense of Mond-Weir in comparison to similarly results proved under other generalized convexity notions.

\section{REFERENCES}

[1] R. P. Agarwal, I. Ahmad, Z. Husain, and A. Jayswal, "Optimality and duality in nonsmooth multiobjective optimization involving V-type I invex functions." J. Inequal. Appl., vol. 2010, p. 898626, 2010, doi: 10.1155/2010/898626.

[2] G. An and X. Gao, "Sufficiency and Wolfe type duality for nonsmooth multiobjective programming problems." Adv. Pure Math., vol. 8, pp. 755-763, 2018, doi: 10.4236/apm.2018.88045.

[3] T. Antczak, "Lipschitz r-invex functions and nonsmooth programming." Numer. Funct. Anal. Optim., vol. 23, pp. 265-284, 2002, doi: 10.1081/NFA-120006693.

[4] T. Antczak, "On G -invex multiobjective programming. Part I. Optimality." J. Global Optim., vol. 43, pp. 97-109, 2009, doi: 10.1007/s10898-008-9299-5.

[5] T. Antczak, "Optimality and duality for nonsmooth multiobjective programming problems with V-r-invexity." J.Global Optim., vol. 45, pp. 319-334, 2009, doi: 10.1007/s10898-008-9377-8.

[6] T. Antczak and A. Stasiak, “( $\Phi, \rho)$ - invexity in nonsmooth optimization." Numer. Funct. Anal. Optim., vol. 32, pp. 1-25, 2011, doi: 10.1080/01630563.2010.526408.

[7] M. Arana-Jimenez, A. Rufian-Lizana, R. Osuna-Gómez, and G. Ruiz-Garzón, "Pseudoinvexity, optimality conditions and efficiency in multiobjective problems: duality." Nonlinear Anal., vol. 68, pp. 24-34, 2008, doi: 10.1016/j.na.2006.10.028.

[8] D. Bhatia and P. Jain, "Generalized $(F, \rho)$-convexity and duality for non smooth multi-objective programs." Optimization, vol. 31, pp. 153-164, 1994, doi: 10.1080/02331939408844012.

[9] F. H. Clarke, Optimization and nonsmooth analysis. United States of America: A WileyInterscience Publication, John Wiley\&Sons, Inc., 1983.

[10] M. A. Hanson and B. Mond, "Further generalization of convexity in mathematical programming." J. Inform. Optim. Sci., vol. 3, pp. 25-32, 1982, doi: 10.1080/02522667.1982.10698716.

[11] A. Jaddar and K. El Moutaouakil, "On weak Pareto optimality for pseudoconvex nonsmooth multiobjective optimization problems." Internat. J. Math. Anal., vol. 7, pp. 2995-3003, 2013, doi: 10.12988/ijma.2013.311276.

[12] V. Jeyakumar, "Equivalence of saddle-points and optima, and duality for a class of nonsmooth non-convex problems." J. Math. Anal. Appl., vol. 130, pp. 334-343, 1988, doi: 10.1016/0022247X(88)90309-5.

[13] V. Jeyakumar and B. Mond, "On generalized convex mathematical programming." J. Austral. Math. Soc. Ser.B, vol. 34, pp. 43-53, 1992, doi: 10.1017/S0334270000007372.

[14] D. S. Kim and S. Schaible, "Optimality and duality for invex nonsmooth multiobjective programming problems." Optimization, vol. 53, pp. 165-176, 2004, doi: 10.1080/0233193042000209435.

[15] H. Kuk, G. M. Lee, and D. S. Kim, "Nonsmooth multiobjective programs with V- $\rho$ - invexity." Indian J. Pure Appl. Math., vol. 29, pp. 405-412, 1998.

[16] G. M. Lee, "Nonsmooth invexity in multiobjective programming." J. Inform. Optim. Sci., vol. 15, pp. 127-136, 1994, doi: 10.1080/02522667.1994.10699173.

[17] X. F. Li and Q. H. L. J. L. Dong, "Lipschitz B - vex functions and nonsmooth programming." J. Optim. Theory Appl., vol. 93, pp. 557-574, 1997, doi: 10.1023/A:1022643129733.

[18] X. Long, "Sufficiency and duality for nonsmooth multiobjective programming problems involving generalized univex functions." J. Syst. Sci. Complex., vol. 26, pp. 1002-1018, 2013, doi: 10.1080/02522667.1994.10699173. 
[19] S. K. Mishra and R. N. Mukherjee, "On generalised convex multi-objective nonsmooth programming." J. Austral. Math. Soc. Ser.B, vol. 38, pp. 140-148, 1996, doi: 10.1017/S0334270000000515.

[20] C. Nahak and R. N. Mohapatra, "Nonsmooth $\rho-(\eta, \theta)$ - invexity in multiobjective programming problems." Optim. Lett., vol. 6, pp. 253-260, 2012, doi: 10.1007/s11590-010-0239-1.

[21] S. Nobakhtian, "Sufficiency in nonsmooth multiobjective programming involving generalized (f, $\rho)$ - convexity," J. Optim. Theory Appl., vol. 130, pp. 359-365, 2006, doi: 10.1007/s10957006-9105-9.

[22] M. V. Stefanescu and A. Stefanescu, "On semi-infinite minmax programming with generalized invexity.” Optimization, vol. 61, pp. 1307-1319, 2012, doi: 10.1080/02331934.2011.563304.

[23] G. J. Zalmai, "Optimality conditions and duality models for generalized fractional programming problems containing locally subdifferentiable and $\rho$ - convex functions." Optimization, vol. 32, pp. 95-124, 1995, doi: 10.1080/02331939508844040.

Authors' addresses

Tadeusz Antczak

Faculty of Mathematics and Computer Science, University of Łódź, Banacha 22, 90-238 Łódź, Poland

E-mail address: tadeusz.antczak@wmii.uni.lodz.pl

Ram Verma

International Publications USA, 1200 Dallas Drive, Suite 912, Denton, TX 76205, USA

E-mail address: ramverna114@yahoo.com 